\title{
The Effects of Physical Activity on Markers of Hepatic Lipid Metabolism during Weight Cycling
}

\section{MATTHEW P. HARRIS, ANDREW SEIJA, RACHAEL HARTKE, MARCUS BREDEN, KAYLEE E. POOLE, and JOSHUA S. WOOTEN}

Exercise Physiology and Biochemistry Lab; Department of Applied Health; Southern Illinois University Edwardsville; Edwardsville, IL

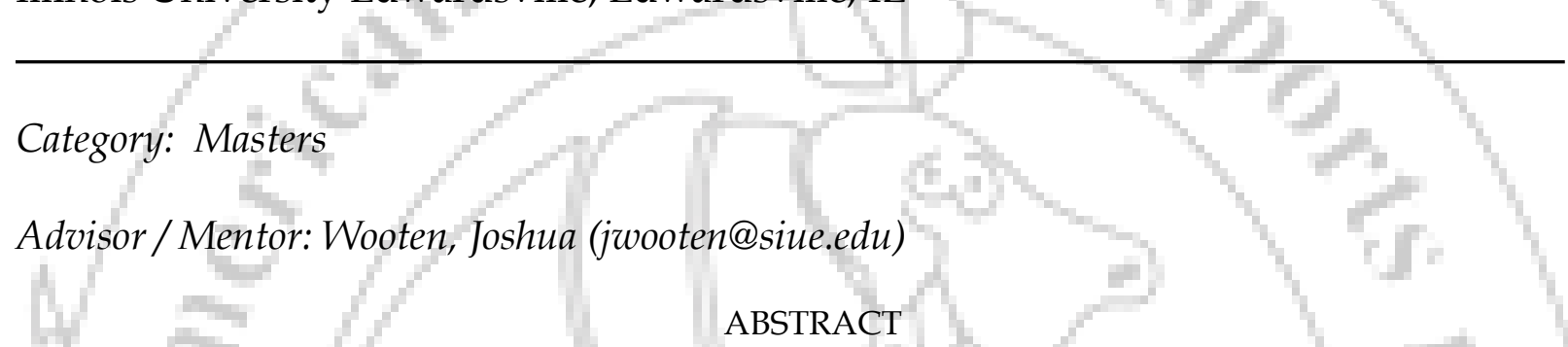

Non-alcoholic fatty liver disease (NAFLD) has emerged as the leading cause of liver disease and develops when the rate of hepatic triglyceride formation exceeds the rate of disposal. Weight loss is often prescribed to treat NAFLD; however, only one in six obese or overweight individuals who lose weight through diet are successful at maintaining weight loss resulting in weight regain (i.e., weight cycling).

PURPOSE: To determine the effect of physical activity on the prevention of hepatic steatosis and expression of lipogenic genes during weight cycling. METHODS: To induce obesity, male C57BL/6 mice were fed a $60 \%$ fat diet for 10 -weeks. Following weight gain, mice were randomly assigned to a $10 \%$ fat diet either with (Diet+PA) or without (Diet) physical activity to induce weight loss for 8 weeks. Physical activity consisted of unrestricted access to running wheels. Following weight loss, the Diet and Diet+PA groups were switched back to a $60 \%$ fat diet for 10 weeks to cause weight regain. The Diet+PA had continued access to physical activity during weight regain. Age-matched lean and obese control mice were fed either a $10 \%$ fat diet (LF) or $60 \%$ fat diet (HF) for the entire 28 weeks of the study. Significant differences $(\mathrm{P}<0.05)$ between groups were identified by one-way ANOVA. RESULTS: Following weight regain, body mass of the Diet+PA was significantly lower than the HF (47.8 vs. $55.3 \mathrm{~g}$ ) and Diet (47.8 vs. $53.9 \mathrm{~g}$ ). No significant difference in body mass was observed between Diet and HF groups. The Diet+PA had significantly lower plasma cholesterol levels compared to HF (230.5 vs. $254.5 \mathrm{mg} / \mathrm{dL}$ ) and Diet (230.5 vs. $271.9 \mathrm{mg} / \mathrm{dL}$ ). In addition, the Diet+PA group had significantly lower total hepatic lipid (23.2 vs. $26.5 \%$ ) when compared with Diet, which was associated with $60 \%, 50 \%$, and $40 \%$ lower expression of lipogenic genes Fasn, Srebp1c, and Chrebp, respectively. No difference was noted between Diet and Diet+PA for the expression of lipogenic genes Scd1 and Acc1. CONCLUSIONS: These data suggests that the continued physical activity during weight cycling resulted in lower weight regain and reduced the accumulation of hepatic lipid by decreased de novo lipogenesis. Overall, the reduced expression of lipogenic related genes might point to a potential protective mechanism that physical activity has on the development of NAFLD during weight cycling.

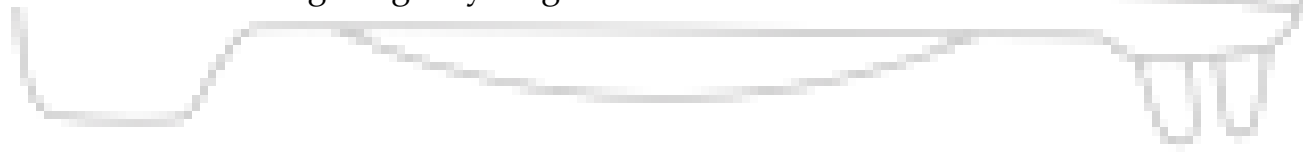

